\title{
Clinico-Radiologic Findings in Primary Cutaneous Extranodal Natural Killer/T-Cell Lymphoma, Nasal Type Mimicking Cellulitis of the Left Arm
}

\author{
Soo Hyun Kim ${ }^{1}$; Hyun Ju Seon ${ }^{1, *}$; Yoo Duk Choi ${ }^{1}$; Sook Jung Yun ${ }^{2}$ \\ ${ }^{1}$ Department of Radiology, Chonnam National University Medical School, Gwangju, South Korea \\ ${ }^{2}$ Department of Dermatology, Chonnam National University Medical School, Gwangju, South Korea \\ ${ }^{*}$ Corresponding author: Hyun Ju Seon, Department of Radiology, Chonnam National University Medical School, Gwangju, South Korea. Tel: +82-622205888, Fax: +82-622264380, E- \\ mail: seonHJ@jnu.ac.kr
}

Received: May 30, 2013; Revised: August 6, 2013; Accepted: August 25, 2013

\begin{abstract}
Extranodal natural killer (NK)/T-cell lymphoma is a very rare and aggressive disease characterized histopathologically by an Epstein-Barr virus (EBV)-positive atypical lymphoid cytotoxic infiltrate, extensive vascular destruction, and prominent tissue necrosis. It commonly shows cutaneous lesions that primarily or secondarily mimic cellulitis at the primary site. We report on a very rare case of extranodal NK/Tcell lymphoma, nasal type of skin/soft tissue, in a 64-year-old man, and describe the radiological findings. The condition was misdiagnosed as cellulitis of the left arm based on initial noninvasive clinical and radiologic work-up.
\end{abstract}

Keywords: Natural Killer T-Cell; Lymphoma; Cellulitis

\section{Introduction}

Natural killer (NK)/T-cell lymphomas are a group of clonal proliferations of cytotoxic lymphocytes of NK or rarely T-cell types, presenting mainly as tumors or destructive lesions in the nasal cavity, maxillary sinuses or palate (1). More rarely, extranodal NK/T-cell lymphomas might become present in other extranodal sites such as the skin, testis, lung, or gastrointestinal tract. Skin involvement as the primary site is rare and is known as primary cutaneous extranodal NK/T-cell lymphoma, nasal type (PC-ENK/T-NT) (2-4). The clinical and radiologic features of PC-ENK/T-NT can mimic cellulitis, panniculitis, or fasciitis. We report a very rare case of PC-ENK/T-NT in the left arm that mimicked cellulitis on initial clinico-radiologic diagnostic work-ups, together with radiologic findings and a review of the literature. To our knowledge, this is the first report of the imaging findings of PC-ENK/T-NT.

\section{Case Presentation}

A 64-year-old man was referred to our hospital due to painful swelling and redness of the left upper arm for three months, which had persisted despite antibiotic treatment at a local clinic (Figure 1 ). The peripheral white blood cell count was elevated to $47 \times 10^{9} / \mathrm{L}$ (52.1\% neutrophils, $32.5 \%$ lymphocytes and $14.3 \%$ monocytes). The hemoglobin level $(152 \mathrm{~g} / \mathrm{L})$ and platelet count $\left(206 \times 10^{9} / \mathrm{L}\right)$ were within normal ranges. The C-reactive protein (CRP) level was mildly elevated to $0.61 \mathrm{mg} / \mathrm{dL}$. The lactate dehydrogenase (LDH) level was $31.06 \mu \mathrm{kat} / \mathrm{L}$. The patient's body temperature was $38.5^{\circ} \mathrm{C}$. Other symptoms such as night sweats and weigh loss (B symptoms) were not prominent. Magnetic resonance imaging (MRI) of the left upper arm of the patient at local clinics showed edematous and inflammatory soft tissue infiltration in the left arm without formation of a definite mass. These findings were suggestive of cellulitis or fasciitis (Figure 2, A and B). On admission, the patient received intravenous antibiotics for three days. He subsequently left the hospital. After seven weeks, the patient returned complaining of newly developed palpable nodules in the left axillary region and aggravated cutaneous lesions in the left upper arm that did not respond to the antibiotic therapy. Left humerus computed tomography (CT) scans at our hospital showed marked soft tissue thickening with edematous changes and ill-defined subcutaneous fatty infiltration in the cutaneous and the subcutaneous layer of the left upper medial arm (Figure 2, C-E). These findings were also suggestive of cellulitis or fasciitis, yet the result of fineneedle aspiration cytology for left axillary lymphadenopathy showed poorly differentiated carcinoma. For further evaluation of the primary site of malignancy, chest CT scans were obtained, which revealed multiple lymphadenopathies with perinodal infiltration in the left axillary area and multiple small and discrete subcutaneous nodules in the anterior chest wall (Figure 3, A and B). A radiologist suggested the possibility of a primary lymphoma, such as a peripheral T-cell lymphoma, rather than a carcinomatous metastasis. An excisional biopsy from the left axillary lymph node and a left upper arm nodule showed extranodal NK/T-cell lymphoma. At the time of skin biop-

Copyright ( 2015 , Tehran University of Medical Sciences and Iranian Society of Radiology. This is an open-access article distributed under the terms of the Creative Commons Attribution-NonCommercial 4.0 International License (http://creativecommons.org/licenses/by-nc/4.0/) which permits copy and redistribute the material just in noncommercial usages, provided the original work is properly cited. 
sy, a dermatologic consultation was performed and the differential diagnosis of the dermatologist was cutaneous lymphoma or vasculitis. The immunophenotyping results were negative for $\mathrm{CD} 3$, positive for $\mathrm{CD} 2$ and $\mathrm{CD} 56$, strongly positive for Epstein-Barr virus (EBV) in situ, and positive for T-cell intercellular antigen (TIA) (Figure 4, A-E). To assess the disease status, positron emission tomography (PET)-CT scans were obtained and revealed multiple nodular lesions in the left axillary area, chest and abdominal walls, upper and lower extremities, and cervical space that showed high fludeoxyglucose (FDG) uptake (Figure 3, C and D). After salvage L-asparagine chemotherapy, all lesions were remarkably improved.

\section{Discussion}

NK/T-cell lymphomas are a group of clonal proliferations of cytotoxic lymphocytes of NK or rarely, T-cell types, with peculiar clinico-pathologic features that present mainly as tumors or destructive lesions in the nasal cavity, maxillary sinuses or palate (1). Rarely, extranodal NK/T-cell lymphoma might become present in other extranodal sites such as the skin, testis, lung, or gastrointestinal tract. This condition is characterized by an EBV-positive atypical lymphoid cytotoxic infiltrate (5), as in the present case. Natural killer/T-cell lymphoma is subcategorized into extranodal NK/T-cell lymphoma, nasal (ENK/T-N) and extranodal NK/T-cell lymphoma, nasal type (ENK/T-NT) by the current World Health Organization (WHO) classification of hematolymphoid tumors $(1,6)$. The ENK/T-NT category accounts for less than $2 \%$ of non-Hodgkin's lymphomas in Europe and North America, but is more frequent in Asia, South America and Central America (7). When the initial site of involvement is the skin, this tumor is known as PC-ENK/T-NT, whereas ENK/T-N lymphoma initially involves the nasal cavity with extension to the skin. The PC-ENK/T-NT lymphoma is rare (2-4). Patients with extranodal NK/T-cell lymphomas show low survival rates and poor responses to treatment (8). In comparison to nasal lymphoma, nasal type lymphoma is clinically less aggressive and more localized, and has a better prognosis (2). Our case showed near-complete remission at six months of treatment with salvage L-asparagine chemotherapy. Clinically, our case mimicked cellulitis and ulceration in the left upper arm, with therapeutic failure of antibiotic treatment, as in a report by Choi et al. (2). Progressive and relatively extensive involvement of cellulitis and relatively deep and large-scale ulceration despite antibiotic therapy could suggest NK-T cell lymphoma to the clinician. In addition to dermatologists, other clinicians should consider this rare disease in such clinical settings with evaluation of the nasal cavity. The CT or MRI findings in PC-ENK/T-NT are not well recognized. In our case, the CT findings were similar to those of cellulitis or fasciitis. However, unlike simple cellulitis or fasciitis, there were mixed findings of multiple discrete cutaneous or subcutaneous nodules, which were similar to the findings for subcutaneous panniculitis-like T-cell lymphomas (9). The nodules in this case showed poorly enhanced peripheral rims and relatively radiolucent central areas in chest CT. In MRI, these lesions showed mixed intermediate to subtle low-signal-intensity areas within the soft tissue swelling and infiltration in a T2-weighted image (T2WI), which was similar to a case of NK/T-cell lymphoma of the face reported by Kim et al. (10). Lesions of simple inflammation, such as cellulitis or fasciitis, usually show high signal intensities in T2W Images. Therefore, the intermediate to subtle low-signal-intensity area in the T2WI is not compatible with simple inflammation and could be an important MRI finding for the differential diagnosis of cutaneous lymphoma from a simple inflammation (10). Familiarity with such CT or MRI findings could provide the radiologist with diagnostic clues about skin or soft tissue involvement of T-cell lymphomas. In FDG-PET imaging, like other T-cell lymphomas, lesions appeared as multiple foci of FDG uptake. Therefore, FDG-PET might
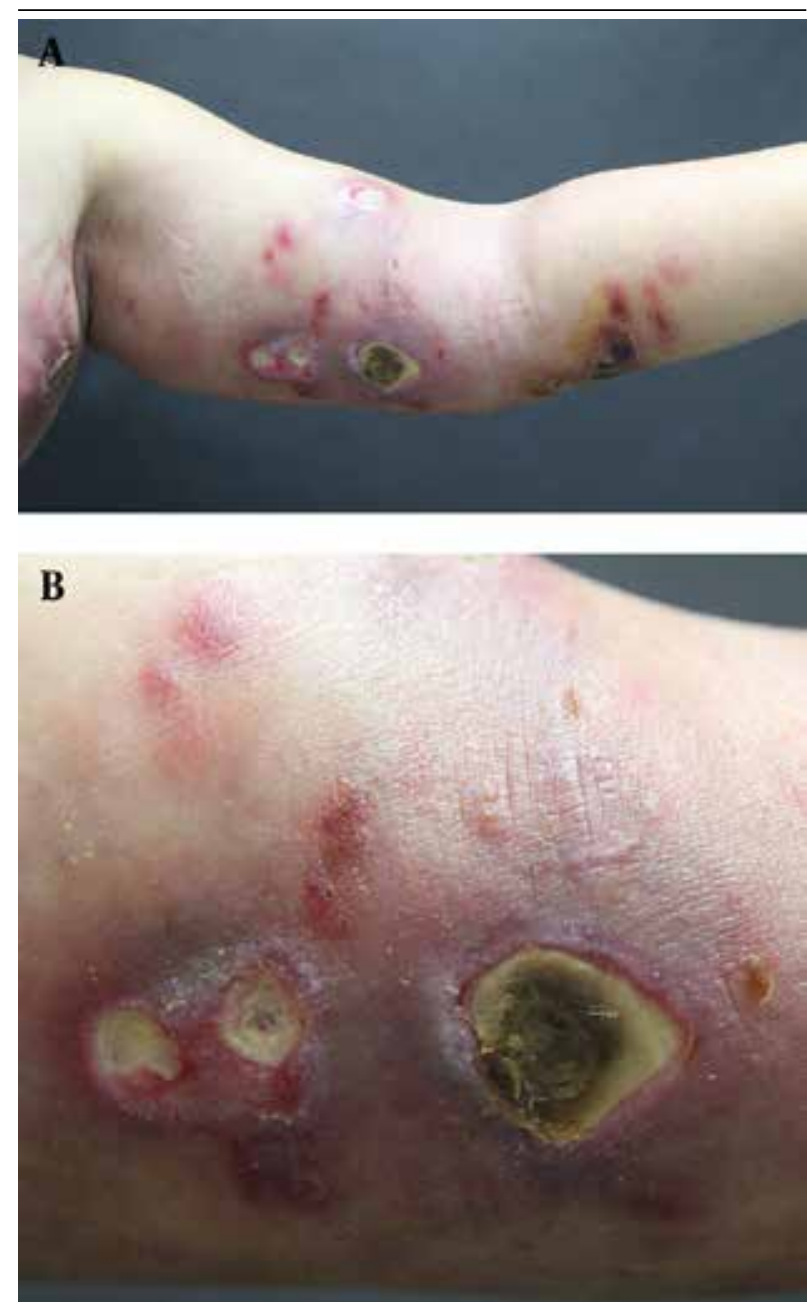

Figure 1. A 64-year-old man with a 3-month history of painful swelling and redness of the left upper arm. Cutaneous manifestation of primary cutaneous extranodal NK/T-cell lymphoma, nasal type. (A, B,) There was erythematous to violaceous swelling, nodules, and ulceration with a crust on the left arm. 
Kim SH et al.
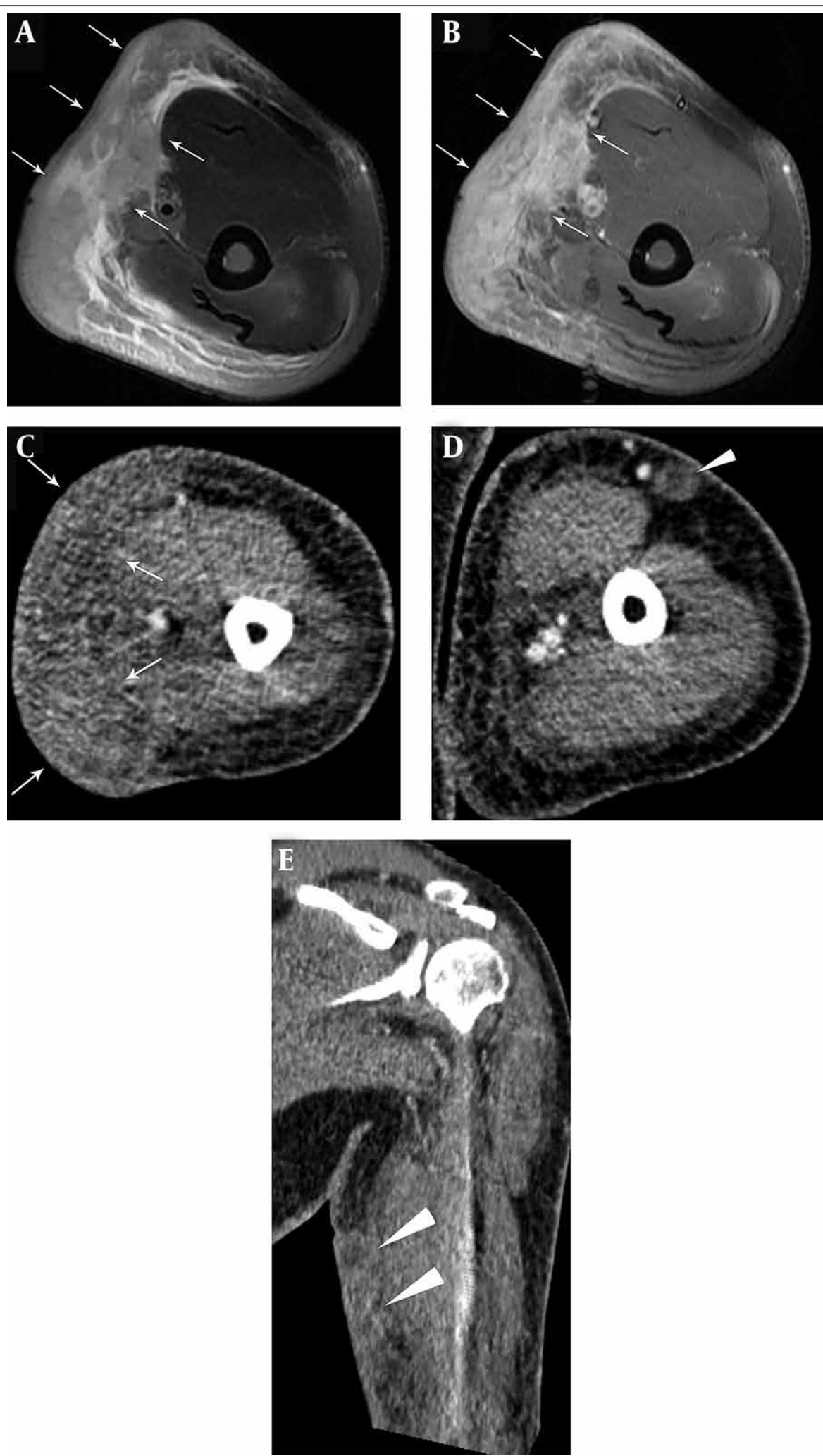

Figure 2. Magnetic resonance imaging and contrast-enhanced computed tomography findings of the left upper arm in a case of primary cutaneous extranodal NK/T-cell lymphoma, nasal type. Fat-suppressed T2-weighted (A) and contrast-enhanced fat suppressed T1-weighted (B) MRI images showed diffuse soft tissue infiltration and subcutaneous edema, with intermediate to subtle low-signal-intensity areas in the T2-weighted image (arrows in A) and heterogeneous enhancement (arrows in B) in the medial aspect of the left upper arm. Enhanced CT images of the left humerus (C-E) also showed diffuse soft tissue infiltration (arrows in C) and mixed subcutaneous nodules (arrowheads in D and E) with poorly enhancing peripheral rims and relatively radiolucent central areas. 

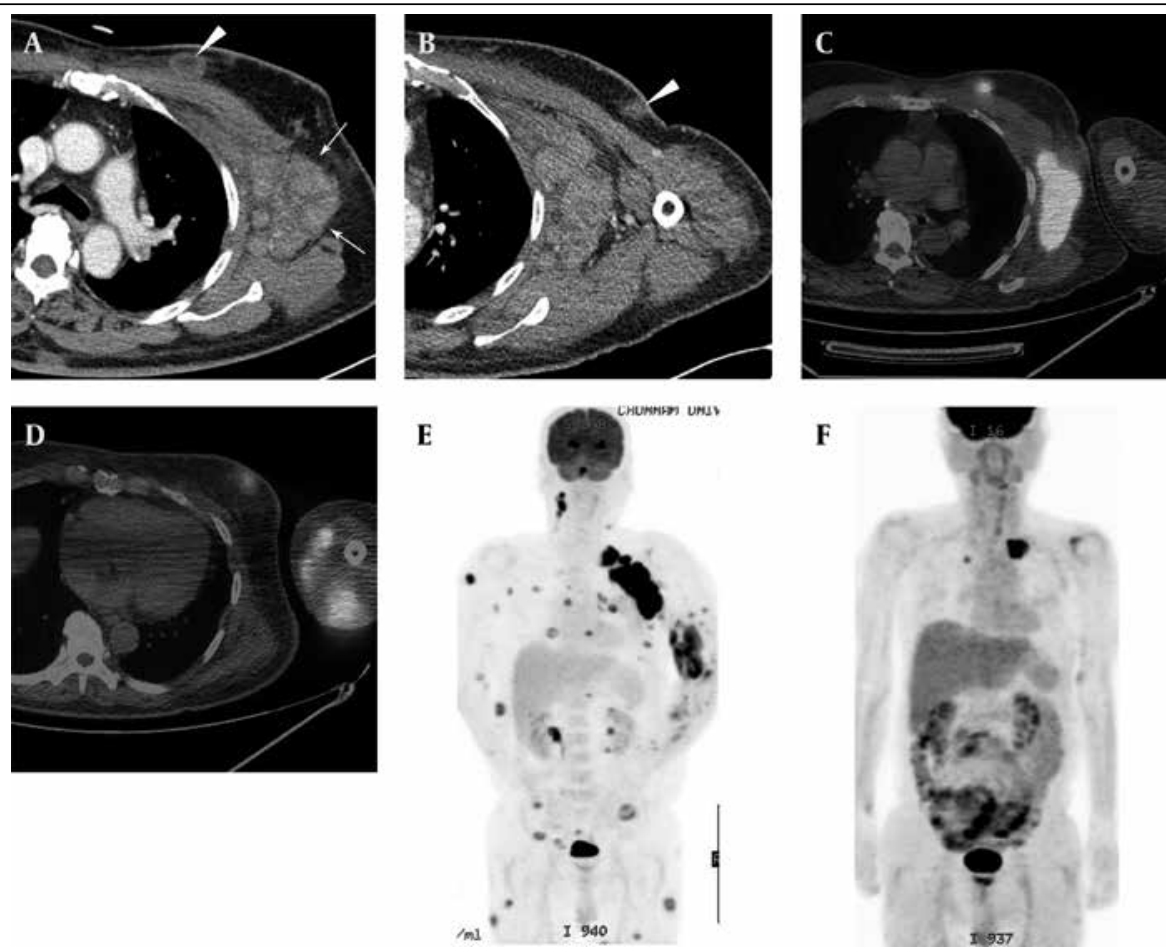

Figure 3. Left axillary lymphadenopathies and cutaneous involvement in a case of primary cutaneous extranodal NK/T-cell lymphoma, nasal type. A and B, Axial contrast-enhanced CT images showed small discrete subcutaneous nodules with poorly enhanced peripheral rims and relatively radiolucent central areas (arrowheads) in the left anterior chest wall, and multiple conglomerated lymphadenopathies in the left axilla (arrows). C and D, These nodules and axillary lymphadenopathies showed high fludeoxyglucose (FDG) uptake in PET-CT images. E, Coronal maximum-intensity-projection PET image showing multiple 18 F-FDG-avid lesions. F, Follow-up PET image after seven months showing regression of multiple 18 F-FDG-avid lesions. The remaining high-FDG-uptake lesion in the left upper lung (arrow) was caused by pneumonic infiltration.
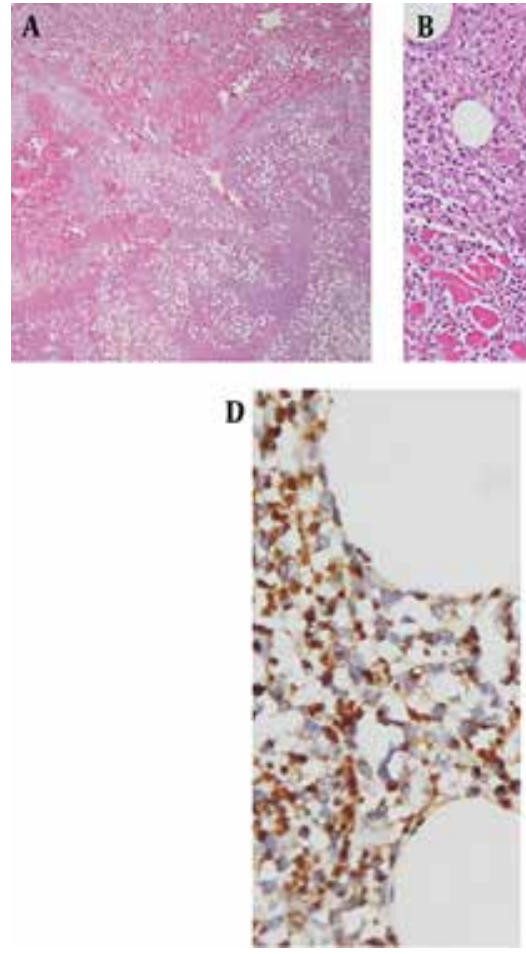
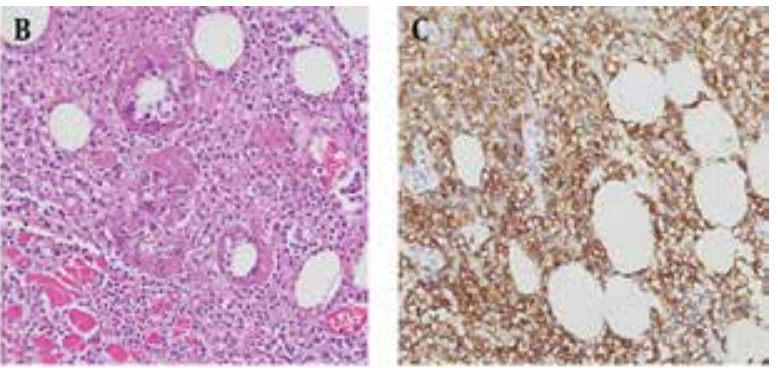

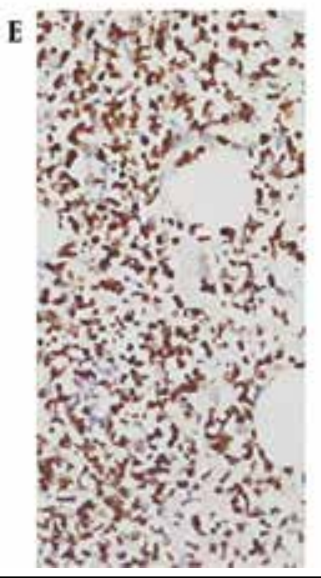

Figure 4. Pathological specimens. A, Atypical lymphoid cell infiltration in the dermis and subcutaneous deep fat layer, with extensive necrosis (H \& E staining, magnification $\times 10$ ). B, Angiodestruction by tumor cells $(\mathrm{H} \& \mathrm{E}$ staining, magnification $\times 200)$. C, Immunohistochemical staining showing that lymphoma cells were positive for CD56. D, Granzyme B. E, Epstein-Barr virus in situ. 
be useful for monitoring of the therapeutic response as well as staging the work-up. After development of left axillary lymphadenopathies, our impression changed from cellulitis or fascitis to a malignant condition. The findings of CT and MRI as well as dermatologic exams could help to differentiate skin or soft tissue lymphoma from benign skin or soft tissue inflammation and lead to early and proper diagnosis and treatment of this aggressive disease.

In conclusion, the clinical and imaging features of PCENK/T-NT are nonspecific and differentiation from skin or soft tissue involvement in benign inflammatory diseases, such as cellulitis and fasciitis, is frequently difficult. Nevertheless, if a patient had skin lesions similar to cellulitis or ulcers that had failed to respond to empirical antibiotics, and imaging findings were not suggestive of benign inflammation, lymphomatous involvement, especially PC-ENK/T-NT, should be considered.

\section{Authors' Contributions}

Hyun Ju Seon and Soo Hyun Kim developed the original idea and wrote the manuscript. Sook Jung Yun contributed to writing the manuscript. Choi YD contributed and reviewed the pathological findings of this case.

\section{Funding/Support}

The authors received no funding or support for this study.

\section{References}

1. Tomonaga M. [Outline and direction of revised WHO classification of Tumors of Haematopoietic and Lymphoid Tissues]. Rinsho Ketsueki. 2009;50(10):1401-6.

2. Choi YL, Park JH, Namkung JH, Lee JH, Yang JM, Lee ES, et al. Extranodal NK/T-cell lymphoma with cutaneous involvement: 'nasal' vs. 'nasal-type' subgroups--a retrospective study of 18 patients. $\mathrm{Br}$ J Dermatol. 2009;160(2):333-7.

3. Pagano L, Gallamini A, Trape G, Fianchi L, Mattei D, Todeschini G et al. NK/T-cell lymphomas 'nasal type': an Italian multicentric retrospective survey. Ann Oncol. 2006;17(5):794-800.

4. Stokkermans-Dubois J, Jouary T, Vergier B, Delaunay MM, Taieb A. A case of primary cutaneous nasal type NK/T-cell lymphoma and review of the literature. Dermatology. 2006;213(4):345-9.

5. Berti E, Recalcati S, Girgenti V, Fanoni D, Venegoni L, Vezzoli P. Cutaneous extranodal NK/T-cell lymphoma: a clinicopathologic study of 5 patients with array-based comparative genomic hybridization. Blood. 2010;116(2):165-70.

6. Willemze R, Jaffe ES, Burg G, Cerroni L, Berti E, Swerdlow SH, et al. WHO-EORTC classification for cutaneous lymphomas. Blood. 2005;105(10):3768-85.

7. Anderson JR, Armitage JO, Weisenburger DD. Epidemiology of the non-Hodgkin's lymphomas: distributions of the major subtypes differ by geographic locations. Non-Hodgkin's Lymphoma Classification Project. Ann Oncol.1998;9(7):717-20.

8. Lee J, Park YH, Kim WS, Lee SS, Ryoo BY, Yang SH, et al. Extranodal nasal type NK/T-cell lymphoma: elucidating clinical prognostic factors for risk-based stratification of therapy. Eur J Cancer. 2005;41(10):1402-8.

9. Kim JW, Chae EJ, Park YS, Lee HJ, Hwang HJ, Lim C, et al. Radiological and clinical features of subcutaneous panniculitis-like T-cell lymphoma.J Comput Assist Tomogr. 2011;35(3):394-401.

10. Kim EY, Kim SS, Ryoo JW, Na DG, Roh HG, Byun HS, et al. Primary peripheral T-cell lymphoma of the face other than mycosis fungoides. Computed tomography and magnetic resonance findings. J Comput Assist Tomogr. 2004;28(5):670-5. 